\title{
Notiz über die Reaktion von Cholesterin mit ס-Methylfurfurol.
}

Von

Carl Neuberg.

(Aus der chemischen Abteilung des Pathologischen Instituts zu Berlin.)

(Der Redaktion zugegangen am 27. Februar 1906.)

In der Festschrift für E. Salkow ski (Berlin 1904, S. 279) habe ich in Gemeinschaft mit Dora Rauchwerger eine Farbenreaktion des Cholesterins beschrieben. Sie besteht darin, daß Cholesterin in alkoholischer Lösung mit einer Spur Rhamnoseoder $\delta$-Methylfurfurollösung versetzt und dann mit konzentrierter Schwefelsäure unterschichtet wird. An der Berührungsfläche entsteht fast sofort ein himbeerfarbener Ring und beim Schütteln unter Kühlung färbt sich die ganze Flüssigkeit himbeerfarben und zeigt, eventuell nach Verdünnung mit Alkohol, einen charakteristischen Absorptionsstreifen, der kurz vor $\mathbf{E}$ beginnt und mit b abschneidet. Der analoge Ausfall der Reaktion mit Substanzen aus der Terpenreihe hat früher die Vermutung nahe gelegt, die Reaktion auf den hydroaromatischen Ring zu beziehen. Nach den Erfahrungen, die seither Guérin ${ }^{1}$ ) bezüglich Farbenreaktionen des Furfurols gemacht hat, wird man sie jedoch auf das Hydroxyl beziehen müssen.

Diese Probe ist durch die Schönheit der Farbe und Schärfe des spektralen Bildes sowie die tagelange Beständigkeit ausgezeichnet. Die früher geäußerte Erwartung jedoch, sie zur Unterscheidung von Cholesterin und Phytosterin verwenden zu können, hat sich nicht bestätigt. Während ein damals benutztes pflanzliches Cholesterin unbekannter Herkunft keine oder höchstens eine angedeutete Reaktion zeigte, konnte nunmehr festgestellt werden, daß Phytosterin aus Baumwollsamenöla) den gleichen Ausfall der Probe gibt wie gewöhnliches Cholesterin.

1) Journ. de Pharm. et Chimie [6], Bd. XXI, S. 14, 1905.

\&) Das Präparat verdanke ich der Güte des Herrn Prof. Salkow ski. 